\title{
Prediction of three-dimensional crutch walking patterns using a torque-driven model
}

\author{
Míriam Febrer-Nafría ${ }^{1}$ \\ Department of Mechanical Engineering \& Research Centre for Biomedical Engineering, \\ Universitat Politècnica de Catalunya \\ miriam.febrer@upc.edu
}

Roger Pallarès-López

Department of Mechanical Engineering \& Research Centre for Biomedical Engineering, Universitat Politècnica de Catalunya

roger.pallares@estudiant.upc.edu

Benjamin J. Fregly

Dept. of Mechanical Engineering, Rice University

fregly@rice.edu

Josep M. Font-Llagunes

Department of Mechanical Engineering \& Research Centre for Biomedical Engineering, Universitat Politècnica de Catalunya

josep.m.font@upc.edu

${ }^{1}$ Corresponding author 


\title{
KEYWORDS
}

Crutch walking, Optimal control, Human motion prediction, Direct collocation

\begin{abstract}
Computational prediction of 3D crutch-assisted walking patterns is a challenging problem that could be applied to study different biomechanical aspects of crutch walking in virtual subjects, to assist physiotherapists to choose the optimal crutch walking pattern for a specific subject, and to help in the design and control of exoskeletons, when crutches are needed for balance. The aim of this work is to generate a method to predict three-dimensional crutch-assisted walking motions following different patterns without tracking any experimental data. To achieve this goal, we collected gait data from a healthy subject performing a four-point non-alternating crutch walking pattern, and developed a 3D torque-driven full-body model of the subject including the crutches and foot- and crutch-ground contact models. First, we developed a predictive (i.e., no tracking of experimental data) optimal control problem formulation to predict crutch walking cycles following the same pattern as the experimental data collected, using different cost functions. To reduce errors with respect to reference data, a cost function combining minimisation terms of angular momentum, mechanical power, joint jerk and torque change was chosen. Then, the problem formulation was adapted to handle different foot- and crutch-ground conditions to make it capable of predicting three new crutch walking patterns, one of them at different speeds. A key aspect of our algorithm is that having ground reactions as additional controls allows to define phases inside the cycle without the need of formulating a multiple-phase problem, thus facilitating the definition of different crutch walking patterns.
\end{abstract}

\section{INTRODUCTION}

Spinal cord injury (SCI), which is commonly caused by falls in the elderly and traffic accidents [1], affects between 250,000 and 500,000 people worldwide each year [2]. Walking impairment after SCI leads to a decreased quality of life and other serious health conditions (e.g., heart disease, high blood pressure) and carries substantial health care costs. Gait restoration of these patients can be partially achieved by powered orthoses and exoskeletons, which usually require the aid of crutches or walkers for balance. This requirement could induce undesirable outcomes if the achieved walking pattern has unwanted features, such as high loads on the shoulders [3-5] or high pressures in the forearm due to skin-crutch contact [6]. To avoid such issues, patients must be trained to learn how to use the exoskeleton together with crutches [7]. The training process is guided by a physiotherapist mainly using visual observation and easily measurable spatiotemporal parameters such as gait speed [8]. Although in some clinical practices a more detailed assessment of a patient's gait pattern is performed to improve training session outcomes [9], an objective method that accounts for patientspecific needs would improve training of these patients.

Computational prediction of the optimal crutch and exoskeleton assisted walking pattern for each patient could be such an objective method to guide patient training. Moreover, it could help in the design and control of those assistive devices, by adapting them to each specific subject. Since SCI patients with the same clinical score can exhibit high motor function variability [10], full-body threedimensional (3D) predictive simulations that explicitly model the arms and crutches could incorporate a patient's functional limitations when seeking to identify the patient's optimal assisted walking pattern. In order to develop this predictive tool, a first stepping stone is to be able to predict various crutch-assisted walking patterns under different optimality criteria. To date, such simulations have been performed using simple models without focusing on specific clinical applications. Previous studies have used optimal control methods to predict swing-through crutch walking using 
simple 2D human-crutch models $[11,12]$ or four-point crutch walking using 3D human models that omitted explicit modelling of the arms and crutches [13]. Furthermore, existing 3D full-body simulations that explicitly modelled the arms and crutches have been driven by measured kinematic data [14] or have tracked measured kinematic data using forward dynamics with torque controllers [15]. Other simulation studies have explored balance when standing with crutches [16] or analysed crutch impact in swing-through crutch gait using different contact formulations [17]. Consequently, researcher efforts to generate three-dimensional crutch walking predictions are still in their early stages.

Beyond crutch walking, previous studies have predicted new walking motions for clinical purposes and rehabilitation treatment design. For instance, Meyer et al. (2016) [18] used a full-body 3D patient-specific neuromusculoskeletal model to predict how a specific individual post-stroke would walk at a faster speed. In an earlier study, Fregly et al. (2007) [19] used a full-body 3D patientspecific skeletal model to predict how a subject with bilateral medial compartment knee osteoarthritis should modify his gait pattern to reduce medial compartment knee loading. Both of these studies were able to validate their walking predictions using additional gait data collected from the patient studied. More recently, Esposito and Miller (2018) [20] used 2D models of 25 virtual patients to predict walking with both passive and active unilateral transtibial prostheses. Furthermore, motion prediction has been used to find the optimal design parameter values for assistive devices: Sreenivasa et al. (2017) [21] identified the optimal stiffness of an ankle-foot orthosis that minimises muscle effort for a pediatric patient, and Mombaur and Ho (2017) [22] designed an assistive device to best support sit-to-stand transfer of geriatric patients. Most of these studies use subject-specific models and multiple shooting [21, 22] or direct collocation [18-20] optimal control methods. These studies are encouraging since they provide objective results that could help with personalised treatment design. However, none of these studies has explored prediction of three-dimensional walking motions with crutches using a full-body model.

This study seeks to develop an optimal control problem formulation capable of predicting different patterns of crutch-assisted walking. To achieve this goal, we collected gait data from a healthy subject performing a four-point non-alternating crutch walking pattern, and developed a 3D torquedriven full-body model of the subject that included both arms, both crutches, and foot- and crutchground contact models. We developed a predictive (i.e., no tracking of experimental data) optimal control problem formulation to predict crutch walking cycles following the same pattern as the experimental data collected, using different cost functions. Then, the problem formulation was adapted to handle different foot-ground and crutch-ground conditions to make it capable of predicting three new crutch walking patterns (alternating four-point, two-point and swing-through patterns), one of them at different speeds.

\section{MATERIALS AND METHODS}

\section{Model development and reference data generation}

\section{Experimental data collection}

Gait data were collected from a single healthy female subject (age 28 yrs., mass $54 \mathrm{~kg}$, height 1.62 $\mathrm{m}$ ) who performed a non-alternating four-point crutch walking pattern (cycle time: $5.75 \mathrm{~s}$, stride length: $0.84 \mathrm{~m}$; Figure 1, left). This crutch walking pattern was chosen since it was different from the crutch walking patterns that were intended to predict, thereby providing a qualitative test case for optimal control crutch walking predictions. We wanted to check if using experimental data of a particular walking pattern to calibrate the model and obtain a first initial guess, we were able to predict new walking patterns. Experimentally measured data included 43 surface marker trajectories from a 16-camera video motion capture system (OptiTrack V100:R2, NaturalPoint Inc., Corvallis, OR, USA), foot-ground reaction forces and torques from two in-ground force plates (AccuGait, AMTI, Watertown, MA, USA), and crutch-ground reaction forces from two instrumented crutches (own-made following the approach used in [23], with 12 strain gauges in each crutch, that measured at $89 \mathrm{~Hz}$ ). Crutch data was interpolated to $100 \mathrm{~Hz}$, as it was the frequency rate for marker trajectories and force plates data. One representative gait cycle was selected for use in all subsequent model development and optimal control problem formulation tasks. 


\section{Skeletal model construction}

A 3D full-body torque-driven skeletal model of the subject using crutches was created starting from a published full-body OpenSim model [24] (Figure 1, centre). The model possessed $n_{q}=37$ degrees of freedom (DOF): 6 DOF between the pelvis and the ground (i.e., translation and rotation), 3 for the lumbo-sacral joint, 3 for each shoulder, 2 for each elbow, 2 for each wrist, 3 for each hip, 1 for each knee, 2 for each ankle, and 1 for each metatarsal joint. Each DOF was associated to a model or joint coordinate $q_{i}\left(i=1, \ldots, n_{q}\right)$ in the order shown above, which formed the $n_{q}$-dimensional vector of generalised coordinates $\boldsymbol{q}$. Each forearm crutch was introduced into the model as a rigid body welded to the corresponding hand segment. Each crutch was added to the model initially with 6 DOF relative motion with respect to the hand. The geometry and mass of each crutch were measured, and its tensor of inertia obtained from simple rigid-body models. To determine constant values for these generalized coordinates, we performed an OpenSim Inverse Kinematic (IK) analysis, calculated the mean value for each translational and rotational coordinate, and replaced each 6 DOF joint with a weld joint consistent with these mean values. Then, we performed a new IK analysis to obtain the experimental joint coordinates used for tracking in the contact model calibration and full dynamically consistent (DC) cycle generation problems.

\section{Contact model calibration}

The skeletal model was enhanced with foot-ground and crutch-ground contact models so that ground reactions could be predicted for new walking motions. The foot-ground contact model consisted of 10 spring and damper units, distributed in four groups on each foot (Figure 1, right). The normal force in each element was generated using a linear spring with nonlinear damping [25], and the spring stiffness, nonlinear damping coefficient, and spring resting length were calibrated for each group of springs. The tangential force in each element was calculated using a simple continuous and differentiable friction model [25], and the coefficient of dynamic friction was calibrated for each group of springs. The crutch-ground contact model consisted of a sphere at the tip of the crutch that could contact a plane representing the ground. The normal force was obtained using a Hertzian elastic point contact model with nonlinear damping [26], and the generalised normal stiffness, hysteresis damping factor, and the sphere radius were calibrated starting from the initial values presented in [17]. The tangential force model was the same as for the foot-ground contact model, and the coefficient of dynamic friction was considered to be 1 . The parameters of the contact models were calibrated solving a direct collocation optimal control problem that tracked the experimental motion and ground reactions simultaneously while adjusting contact model parameter values, which were assumed to be the same on both sides [27]. Mean root-mean-square errors (RMSE) for ground reactions obtained from contact models compared to experimental measurements were $5.53 \mathrm{~N}$ for forces and $6.27 \mathrm{Nm}$ for moments (Table 1).

\section{[TABLE 1]}

\section{Dynamically consistent reference data generation}

Once the foot-ground and crutch-ground contact model parameters were calibrated, we used the fullbody skeletal model with crutches to generate a DC crutch-walking motion that closely reproduced the experimentally measured motion and ground reactions. To generate this motion, we formulated and solved an optimal tracking problem whose cost function minimised the weighted sum of two error terms: 1) errors between model and experimental joint angles, and 2) errors between model and experimental ground reactions from the feet and crutches. Residual forces and torques acting on the model pelvis were limited to be within a specified range using path constraints. The tracking problem also predicted the ground reactions under the right foot for approximately the first $15 \%$ of the gait cycle, since the ground reactions under both feet for an entire gait cycle cannot be measured using only two force plates. Periodicity was imposed for ground reaction forces and moments with a tolerance of $\pm 20 \mathrm{~N}$ and $\pm 20 \mathrm{Nm}$, respectively. A full DC cycle was obtained with the following mean RMSE with respect to original experimental data: $1.67^{\circ}$ and $1.28 \mathrm{~cm}$ for rotational and translational coordinates, respectively; and $7.71 \mathrm{~N}$ and $5.44 \mathrm{Nm}$ for ground reaction forces and moments, respectively. The resulting DC crutch-walking motion produced by the model was used as reference data for subject optimal control crutch-walking predictions (Figure 2, top box).

[FIGURE 2] 


\section{Optimal control formulation for crutch-walking predictions}

\section{Implicit dynamics}

Crutch-walking prediction problems were formulated as a direct collocation optimal control problems (OCP) using implicit skeletal dynamics, as implicit dynamics has been shown to works better than does explicit skeletal dynamics when solving direct collocation OCPs involving human movement [28]. The OCP solver used in this work, GPOPS-II [29], does not handle implicit dynamics and requires the use of explicit dynamics for dynamic constraints. We addressed this limitation by adding an implicit form of skeletal dynamics as path constraints, adding joint jerk or torque derivative as additional controls, and using derivative relationships (Eq. 1) to define the required explicit dynamics [18]. To make the controls unique, we included a joint jerk or torque derivative regularisation term in the cost function $[18,30]$. The torque derivative criterion is similar to the joint jerk criterion [31], and both criteria have been used for human walking prediction in previous studies [18,32-34]. States and controls varied depending on the regularisation term included in the cost function. When minimising joint jerk, states were joint coordinates, joint velocities and joint accelerations; and controls were joint jerk and joint torque. When minimising joint torque change, states were joint coordinates, joint velocities and joint torques; and controls were joint acceleration and joint torque change (Table 2). Therefore, as an example, the dynamic constraints in the problem for the case of minimising joint jerk were:

$$
\left[\frac{d \boldsymbol{q}}{d t}, \frac{d \dot{\boldsymbol{q}}}{d t}, \frac{d \ddot{\boldsymbol{q}}}{d t}\right]^{T}=[\dot{\boldsymbol{q}}, \ddot{\boldsymbol{q}}, \ddot{\boldsymbol{q}}]^{T}
$$

where $\boldsymbol{q}, \dot{\boldsymbol{q}}, \ddot{\boldsymbol{q}}, \dddot{\boldsymbol{q}}$ stand for the vector of generalised joint coordinates, velocities, accelerations and jerks, respectively.

The skeletal equations of motion from OpenSim were included implicitly as algebraic path constraints. An Inverse Dynamics (ID) analysis was performed at each iteration using the OpenSim $\mathrm{C}++$ API (version 3.3), and the system kinematic state was used to calculate the net forces and torques applied at each DOF (which included the 6 residual loads acting on the pelvis $\boldsymbol{R}_{\text {pelvis }}$, and the $n_{q}-6$ joint torques $\left.\boldsymbol{\tau}_{I D A}\right)$. Path constraints limited the residual loads to be within a specific tolerance $\boldsymbol{\varepsilon}$; and if joint torques $\boldsymbol{\tau}$ were variables of the OCP, they were forced to be the same (within a specific tolerance $\boldsymbol{\varepsilon}^{\prime}$ ) as joint torques obtained from the ID analysis:

$$
\begin{gathered}
-\boldsymbol{\varepsilon} \leq \boldsymbol{R}_{\text {pelvis }}(\boldsymbol{q}, \dot{\boldsymbol{q}}, \ddot{\boldsymbol{q}}) \leq \boldsymbol{\varepsilon} \\
-\boldsymbol{\varepsilon}^{\prime} \leq \boldsymbol{\tau}_{I D A}(\boldsymbol{q}, \dot{\boldsymbol{q}}, \ddot{\boldsymbol{q}})-\boldsymbol{\tau} \leq \boldsymbol{\varepsilon}^{\prime}
\end{gathered}
$$

Additional controls were added representing ground reactions for two reasons: (1) these controls were used as inputs for the ID analysis at each iteration, improving convergence because they facilitated a solution that was dynamically consistent; and (2) these controls were forced to be zero during swing phase for each foot and crutch, which permitted us to formulate a problem that had multiple phases, without having explicitly multiple phases in GPOPS-II, simplifying the problem and reducing computation time. Additional path constraints ensured that ground reactions resulting from contact models were equal to ground reactions controls. To define the end of a cycle, stride length was used to impose the distance between initial and final position of the pelvis, each foot midpoint, and each crutch tip in the anterior-posterior direction. Finally, lateral distances between feet and crutches were limited to avoid lateral crossing, and velocity of some specific points (midpoint for feet and tip for crutches) during stance phase was bounded in order to avoid sliding.

[TABLE 2]

\section{Cost function comparison}

Different optimality criteria that have been used to predict human walking with skeletal models were used to predict crutch walking. The optimality criteria considered in this study were minimising mechanical power, which can be considered to be a measure of energy consumption when using torque-driven models $[33,35]$, angular momentum, which has been found to be small for human walking [36, 37], and kinetic energy [32]. The three optimality criteria were combined with regularisation terms that minimised squared joint jerk (i.e., time derivative of joint acceleration) or torque change (i.e., time derivative of joint torque), thereby making the control solution unique. The 
general cost function can be described as:

$$
J=\int_{t_{0}}^{t_{f}}\left(J_{o p t}(\boldsymbol{x}, \boldsymbol{u})+0.01 J_{\text {reg }}(\boldsymbol{u})\right) d t
$$

where $t_{0}$ and $t_{f}$ are the initial and final simulation times, respectively, $\boldsymbol{x}$ is the vector of states, and $\boldsymbol{u}$ is the vector of controls. Three different optimality terms $J_{o p t}(\boldsymbol{x}, \boldsymbol{u})$ were used: (1) the sum of squared mechanical power, computed for each relative coordinate (i.e., not for the absolute pelvis translation and rotation coordinates), $\sum_{i=7}^{n_{q}}\left(\dot{q}_{i} \tau_{i-6}\right)^{2}$, being $\dot{q}_{i}$ the $i^{\text {th }}$ component of the vector of joint velocities $\dot{\boldsymbol{q}}$, and $\tau_{i-6}$ the $(i-6)^{t h}$ component of the vector of joint torques $\boldsymbol{\tau}$; (2) the sum of the squared norms of the local angular momenta, $\sum_{i=1}^{n_{b}}\left\|\boldsymbol{L}_{i}\right\|^{2}$, being $n_{b}$ the number of rigid bodies in the model and $\boldsymbol{L}_{i}$ the local angular momentum at the centre of mass of the $i^{\text {th }}$ body of the model; and (3) the squared value of the model kinetic energy, $T^{2}$. Two different regularisation terms $J_{\text {reg }}(\boldsymbol{x}, \boldsymbol{u})$ were considered: (1) the sum of squared joint jerks, $\sum_{i=1}^{n_{q}} \dddot{q}_{i}^{2}$, being $\dddot{q}_{i}$ the $i^{\text {th }}$ component of the vector of joint jerks $\dddot{\boldsymbol{q}}$; and (2) the sum of squared joint torque changes, $\sum_{i=1}^{n_{q}-6} \dot{\tau}_{i}^{2}$, being $\dot{\tau}_{i}$ the $i^{\text {th }}$ component of the vector of joint torque change $\dot{\boldsymbol{\tau}}$. This resulted in a total of six cost functions that were compared. All cost function terms were scaled to be of similar magnitude. To give more importance to the optimality criteria, a weight of 0.01 was placed on the term that minimised joint jerk or torque change.

Using the reference gait cycle as an initial guess and the different cost function formulations, new crutch-walking motions that followed the same four-point non-alternating gait pattern were predicted without tracking any experimental data (Figure 2, middle box). Stride length and phase durations were imposed from the experimental data to obtain predicted crutch-assisted walking motions comparable to the reference motion. To assess the results and choose the best cost function for crutch walking prediction, root mean square errors (RMSE) between predicted and the reference quantities were calculated for joint coordinates and ground reactions. Differences in joint ranges of motion (ROM) were also computed.

\section{Crutch walking pattern predictions}

To develop a method that can predict any crutch-assisted walking pattern, we made several modifications to the previous problem formulation. The walking cycle was parameterised based on swing phase duration and stride length. We assumed that swing duration was the same for feet and crutches for both the right and left sides. Duration of multiple support phases between consecutive swing phases were also considered to be the same. Three new walking patterns were predicted: (1) four-point alternating gait, (2) two-point (or reciprocal) gait, and (3) swing-through gait (Figure 2, bottom box). For each walking pattern, the sequence of swing phases was as follows: (1) four-point alternating gait: right crutch, left leg, left crutch, right leg; (2) two-point gait: right crutch and left leg, left crutch and right leg; and (3) swing-through gait: both crutches, both legs ahead of the crutches [38] (Figure 3, right). The initial position, which was added as an endpoint constraint, was also pattern-dependent (Figure 3, left). Swing duration and stride length were known values, taken from the measured motion $(0.96 \mathrm{~s}$ and $0.84 \mathrm{~m}$, respectively). Variations from the first new walking pattern (four-point alternating gait) were predicted for faster and slower speeds by modifying swing duration or stride length (Table 3). Both quantities, swing duration and stride length, were known and imposed in the problem formulation: swing duration was used to define when ground reactions needed to be zero, and stride length was imposed in the endpoint constraints, as explained previously. To assess the results between gait patterns, joint coordinates, joint torques, and ground reactions were compared against results from literature, and ground reactions were compared among gait patterns. Moreover, to assess the results from variations within the same gait pattern, joint coordinates were compared against results for the prediction with the initial values for swing duration and stride length. All optimisations were performed using a Dell Precision Tower 5810 (Intel(R) Xeon(R) CPU E5-1620 v3 @ 3.50GHz, 16.00 GB RAM).

[FIGURE 3]

[TABLE 3] 


\section{RESULTS}

All six cost functions converged for the four-point non-alternating gait, with stride length and swing durations imposed from experimental data. Convergence was better (less number of iterations and less computation time) for the case of minimising mechanical power and joint jerk, closely followed by the case of minimising angular momentum and torque change (Table 4). Convergence was worse (up to 10 times more iterations and computation time) when minimising kinetic energy and joint jerk. Overall, minimising angular momentum showed less error with respect to the reference motion than minimising mechanical power or kinetic energy. Mean RMSE for all angular coordinates was lower when minimising angular momentum and joint jerk $\left(6.30^{\circ}\right)$, while mean difference of ROM for all angular coordinates was lower when minimising angular momentum and torque change $\left(7.26^{\circ}\right)$. With jerk minimisation, differences in joint coordinates ROM with respect to reference values were larger $\left(7^{\circ}\right.$ to $\left.18^{\circ}\right)$ than with torque change minimisation $\left(5^{\circ}\right.$ to $\left.15^{\circ}\right)$. In all cases, ROMs were lower for the solution than for the reference motion, so the larger the difference in ROM, the smaller the ROM was for that group of coordinates. In the case of torso coordinates (i.e., lumbar joint), RMSE was lower for minimisation of mechanical power and joint jerk $\left(5.96^{\circ}\right)$, and ROM difference was lower for minimisation of kinetic energy and torque change $\left(7.30^{\circ}\right)$. Regarding ground reactions, minimising mechanical power and joint jerk was the cost function with lowest mean RMSE for normal forces $(40.2 \mathrm{~N})$, while minimising angular momentum and torque change was the cost function with lowest mean RMSE for tangential forces $(12.67 \mathrm{~N})$. Qualitatively, minimising joint jerk led to smoother coordinates than minimising torque change, while minimising torque change led to smoother ground reactions than minimising joint jerk.

\section{[TABLE 4]}

According to these results, a multi-term cost function was considered, taking for each part of the body the optimisation criterion for which best results in terms of error were achieved. The proposed cost function was minimising angular momentum of upper and lower limb segments, mechanical power associated to lumbar and lower limb joint coordinates, and both joint jerk and torque change. In this case, states were joint coordinates, joint velocities, joint accelerations and joint torques; and controls were joint jerk and joint torque change (Table 2). Using the multi-term cost function, the computation time and number of iterations increased when compared to the previous formulations, but the results in terms of RMSE and differences in ROM with respect to the reference motion were the best overall (Table 4, last column). Pelvis tilt, pelvis rotation, lumbar extension and hip flexion were well predicted, both in shape and in ROM, and RMSEs for these coordinates were between $3.3^{\circ}$ and $6.4^{\circ}$ (Figure 4). On the other hand, hip rotation, lumbar bending, arm flexion and wrist flexion followed the tendency of experimental data, but ROMs were reduced (e.g., $30^{\circ}$ less ROM for arm flexion). Regarding ground reactions, normal forces followed the tendency of reference data, but the computed forces were smoother than the experimental ones, especially foot-ground reactions (Figure 5).

\section{[FIGURE 4]}

\section{[FIGURE 5]}

Having the walking cycle parameterised based on the swing phase duration and stride length, three new walking patterns (for which no experimental data were available) were predicted: four-point alternating, two-point and swing-through crutch gait patterns. Four-point alternating pattern converged when using the multi-term cost function, but two-point and swing-through patterns did not. For that reason, the cost functions for which convergence was better -minimising mechanical power and joint jerk, and minimising angular momentum and torque change (Table 4)- were used to predict these new patterns. It was found that the three new patterns converged when minimising angular momentum and torque change. These results were considered to compare the three new crutch-assisted walking patterns. Maximum values for vertical foot-ground reactions were around $50-60 \%$ body weight (BW) for four-point gait, almost $80 \% \mathrm{BW}$ for two-point gait, and around $50 \%$ BW for swing-through gait (Figure 6). In the case of crutches, the maximum values for vertical reactions were $35 \% \mathrm{BW}$ for four-point gait, $30 \%$ for two-point gait, and $50 \% \mathrm{BW}$ for swing-through gait (Figure 6). Shoulder angles were similar for four-point gait and two-point gait patterns, in terms of ROM and maximum and minimum values (Figure 7, top). Regarding swing-through gait pattern, shoulder flexion-extension presented higher symmetry than the other shoulder coordinates. Shoulder flexion and adduction torques were higher for the swing-through gait pattern. For example, maximum value for shoulder flexion torque was $5.4 \% \mathrm{BW} * \mathrm{H}$ (i.e., percentage of body weight times height), compared to $1.38 \% \mathrm{BW} * \mathrm{H}$ for two-point gait and $2.9 \% \mathrm{BW} * \mathrm{H}$ for four-point gait (Figure 
7, bottom).

[FIGURE 6]

[FIGURE 7]

Our formulation allowed to predict crutch walking cycles at different speeds. For the four-point alternating crutch gait pattern, predictions at higher and lower speeds were obtained by modifying initial stride length or swing duration values (Table 3). The cost function used was the multi-term cost function. Convergence was better when only swing duration was modified, without varying stride length (36-39 iterations); while varying stride length, without varying swing duration, required much more iterations (298-363). Considering root mean square differences (RMSD) for all angular coordinates with respect to the movement at initial speed, the closest solution was the one with low swing duration and initial stride length (mean RMSD was $2.4^{\circ}$ ), while the solution with larger differences was the one with high stride length and initial swing duration (mean RMSD of $5.9^{\circ}$ ). For constant swing duration, differences in some coordinates were observed when stride length was modified (Figure 8). For the case of low speed (that is, low stride length), the larger difference was found in lumbar joint (mean RMSD of $5.71^{\circ}$ ). In this case, the torso was less inclined (i.e., sum of pelvis tilt and lumbar extension was lower in absolute value) and hip flexion was lower during all cycle compared to the movement at initial speed. For the case of high speed (that is, high stride length), the larger difference was obtained for lower limb joints (mean RMSD of $6.6^{\circ}$ ). In this case, hip flexion was lower during swing phase for right leg and higher during swing phase for left leg compared to the movement at initial speed. Moreover, elbow flexion was higher during almost all cycle time.

[FIGURE 8]

\section{DISCUSSION AND CONCLUSIONS}

The aim of this work was to generate a method to predict three-dimensional crutch-assisted walking motions following different patterns without tracking any experimental data. For that, different cost functions were tested, comparing the obtained predictive simulations with experimental data (joint coordinates and ground reactions) from a healthy subject following a four-point non-alternating crutch gait pattern. To reduce errors with respect to reference data, a cost function combining minimisation terms of angular momentum, mechanical power, joint jerk and torque change was chosen (Table 4). This cost function was used to predict four-point alternating crutch gait at the same speed as the experimental conditions and at a higher and lower speeds. However, this cost function did not converge for two-point and swing-through crutch gait patterns, which were predicted minimising angular momentum and torque change. The fact that not all patterns converged for the cost function that gave better results for four-point non-alternating gait may indicate that the optimality criteria are pattern-dependent. A key aspect of our algorithm is that having ground reactions as additional controls allows to define phases inside the cycle without the need of formulating a multiple phase problem, and facilitating the definition of different crutch walking patterns. Small variations to predict more weight bearing on one side than the other or having only one crutch are easy to implement, giving a wide margin of applications to this formulation. To our knowledge, no studies have yet predicted three-dimensional full-cycle crutch walking patterns using a full-body model. Moreover, there are few studies where novel gait motions are predicted without tracking any experimental data [39].

The combination of terms chosen for the cost function (minimising angular momentum of upper and lower limbs, mechanical power associated to lumbar and lower limbs joint coordinates, and both joint jerk and torque change) presented overall better results in error terms when compared to the six initial cost functions for the four-point non-alternating crutch gait pattern (Table 4). However, there were not large differences in errors among cost functions (e.g., the mean RMSE for full body angular coordinates varies from $6.30^{\circ}$ to $9.19^{\circ}$, with a difference of less than $3^{\circ}$ ), and some solutions were very similar in terms of joint angles evolution. For instance, regarding ground reaction normal forces (Figure 5), the difference between the lower and the largest RMSE was $18 \mathrm{~N}$, that is, only a $3 \%$ of the maximum value for experimental normal forces. As different optimality criteria lead to similar solutions, it seems that the solution depends more on how the problem is formulated (in terms of constraints and initial guess, for example) than on the particular criterion used in each case. It is not clear how these criteria are related among them or with the task of crutch walking [40, 41]. The fact that the proposed formulation has predicted correctly four-point gait (alternating and non- 
alternating) does not imply that it corresponds to the physiologic criteria that we follow when walking with crutches. More research is needed to find these criteria. In our predictive simulations, we found that qualitatively joint jerk minimisation produced smoother joint coordinates, while torque change minimisation produced smoother ground reactions. The minimisation of both variables at a time produced coordinates and ground reactions that were not as smooth as when they were minimised separately, but looked qualitatively more realistic. We have not found studies that minimise at the same time joint jerk and torque change, as they are considered to be comparable criteria [31, 33], although they produce different motions for gait [32] or arm reaching tasks [4244].

Results for the three new crutch walking patterns (four-point alternating, two-point, swing-through) were consistent with experimental results from previous studies on crutch locomotion. Regarding ground reactions, in swing-through gait pattern the weight is distributed in a similar way between feet and crutches, while in four-point and two-point gait patterns, more weight is supported by the feet [38]. In our results, the maximum values for normal forces were around $50 \% \mathrm{BW}$ for feet and crutches in swing-through gait; $60 \% \mathrm{BW}$ for feet and 35\% BW for crutches in the case of four-point pattern; and $80 \% \mathrm{BW}$ for feet and $30 \% \mathrm{BW}$ for crutches in the case of two-point pattern (Figure 6). Moreover, feet normal forces were higher in two-point pattern (maximum values around $80 \% \mathrm{BW}$ ) than in four-point pattern (maximum values around $60 \% \mathrm{BW}$ ). This result is in agreement with [45], where plantar pressures in normal walking, four-point crutch walking and two-point crutch walking were measured and compared. Regarding shoulder coordinates, shoulder flexion-extension was inside the maximum and minimum values extracted from $[5,6]$, and followed a similar pattern for four-point and two-point gaits (Figure 7). In the case of swing-through, the results were almost symmetric (e.g., maximum difference between right and left shoulder flexion was around $4^{\circ}$ ), although symmetry was not imposed, which indicates that the movement of both arms and legs is parallel $[46,47]$. Conversely, the ROM and mean value for shoulder abduction-adduction, though similar for all three walking patterns (ROM varied from $6.56^{\circ}$ to $15.41^{\circ}$, and mean values varied from $-27.37^{\circ}$ to $-15.45^{\circ}$ ), were not inside the maximum and minimum values extracted from $[5,6]$. These differences may be due to the large variability presented in crutch walking among subjects [6]. Regarding shoulder torques, swing-through gait presented the highest values for shoulder flexion (a maximum of $5.4 \% \mathrm{BW}^{*} \mathrm{H}$ ) and abduction (a maximum of $2.17 \% \mathrm{BW}^{*} \mathrm{H}$ ) moments, which is consistent with the fact that this pattern requires considerable upper body strength to support the entire body weight $[38,46]$. However, the values obtained in our simulations are higher than those extracted from [5].

Results for the different variations of four-point alternating gait were reasonable. The initial guess used was the solution with initial stride length $(0.84 \mathrm{~m})$ and initial swing duration $(0.96 \mathrm{~s})$, so it was expected that much less iterations were needed for the solutions with the initial stride length, compared with the solutions with the initial swing duration and modified stride length. For the initial duration and lower stride length, the strategy followed by the solution to reduce stride length was to incline less the torso (on average $5.65^{\circ}$ less inclined) and to reduce shoulder flexion and elbow flexion (for the right side, $5.92^{\circ}$ and $2.69^{\circ}$ less on average for shoulder and elbow flexion, respectively) (Figure 8). Conversely, for the initial duration and higher stride length, the strategy followed by the solution to increase step length was to increase hip flexion (for the left side, the maximum value for hip flexion was $3.01^{\circ}$ higher than for the initial step length). Although this was not done symmetrically, as hip flexion increased for the left leg but decreased for the right leg, the variations of both at the same time produced a higher stride length. A longer step length is related with more hip flexion [48] and, in crutch walking, step length is also related with shoulder and elbow flexion. This is in agreement with our predictive crutch walking simulations, where reducing shoulder and elbow flexion produced gaits with reduced stride length, and increasing hip flexion produced gaits with increased stride length.

This study possesses some limitations that need to be further investigated in order to improve the algorithm. Only a single walking cycle for one subject and one type of walking pattern was collected, and we decided to use manual tuning of each term in the cost function [37, 40], instead of solving an inverse optimal control problem to find the weights for each term $[49,50]$. Moreover, we investigated only terms that are used for normal walking prediction, that may not be exactly the ones representing the physiological criteria for crutch locomotion prediction. Therefore, more research is needed regarding the cost function formulation for crutch-assisted locomotion. Another limitation is that the process of generating a first initial guess for each crutch walking pattern was manual. For the different walking patterns, the same formulation (cost function and constraints) could converge or not depending on the initial guess. Since this is a common limitation in direct collocation methods, as the convergence relies on having good initial guesses [39], more research is needed on how to 
objectively define this initial guess. Moreover, the obtained solutions could be local minima, because for different combinations of IPOPT and mesh tolerances that we tested (for the four-point nonalternating gait pattern), each cost function converged to different solutions. In future investigations we will start from different initial guesses in order to try to overcome this issue. Finally, we did not perform any sensitivity analysis to check how do the foot-ground and crutch-ground contact model parameter values affect the results (especially the radius and the stiffness parameters of the crutchground contact model), but we think that the methods and the results presented here are still valid and valuable.

Future work will seek to improve the problem formulation so that these methods can be used to predict crutch and exoskeleton assisted walking patterns, with the aim of personalising an exoskeleton controller to the unique needs of individual SCI subjects [51]. Now that the proposed optimal control method has been developed, we plan to collect additional experimental data (including different subjects using different crutch-walking patterns) to improve the accuracy of our prediction process. With new experimental data, we will have more information to investigate if the best cost function is pattern-dependent. Regarding the problem formulation, different aspects need more research. First, we will add gait cycle duration and stride length as free parameters to the optimal control problem formulation, allowing us to predict the optimal crutch walking speed for a specific subject. Second, we will also investigate other cost function formulations that might better represent crutch walking. Regarding our final application, the methods developed in this study will be used to predict computationally the optimal crutch and exoskeleton assisted walking pattern for SCI patients. The model will be extended to include the subject's functional limitations, and the problem will be adapted to predict impaired gait, which is much more complex than healthy gait.

The authors foresee other potential applications based on the methods presented in this study. For example, having an algorithm that allows to predict different crutch walking patterns is a first stepping stone toward developing a predictive tool to assist physiotherapists to choose the best crutch and exoskeleton assisted walking pattern for a specific subject to optimise gait training. Moreover, this approach could also be very useful for other researchers to study different biomechanical aspects of crutch walking, as it could overcome some limitations that existing studies of crutch walking in gait analysis laboratories declare, such as difficulty in recruiting subjects or limitation in the number of tests that can be performed [52-55].

\section{ACKNOWLEDGMENTS}

We thank Fernando Salvucci and Víctor A. Carmona-Ortiz for helping with the instrumentation and calibration of the crutches. This work has been supported by the Spanish Ministry of Economy and Competitiveness (MINECO) along with the European Regional Development Fund (ERDF) under project DPI2015-65959-C3-2-R, and by the BBVA Foundation under a 2018 Leonardo Grant for Researchers and Cultural Creators. 


\section{TABLES}

Table 1: Calibrated parameters for foot-ground [25] and crutch-ground [26] contact models. Symmetry was imposed between right and left feet, and right and left crutches. Each group of springs for the foot-ground contact model is shown in Figure 1, right.

\begin{tabular}{|c|c|c|c|c|}
\hline Foot-ground contact model parameter & Group 1 & Group 2 & Group 3 & Group 4 \\
\hline Spring stiffness $[\mathrm{N} / \mathrm{m}]$ & 2605 & 2886 & 4014 & 3563 \\
\hline Damping coefficient $[\mathrm{s} / \mathrm{m}]$ & 45.75 & $1.45 \cdot 10^{-4}$ & 1.44 & 1.91 \\
\hline Spring resting length $[\mathrm{cm}]$ & 0.75 & -0.55 & -1.50 & -1.39 \\
\hline Coefficient of dynamic friction [-] & 0.18 & 0.09 & 0.15 & 0.46 \\
\hline Crutch-ground contact model parameter & Tip & & & \\
\hline Generalised normal stiffness $\left[\mathrm{N} / \mathrm{m}^{3 / 2}\right]$ & $9.35 \cdot 10^{3}$ & & & \\
\hline Hysteresis damping factor $\left[\mathrm{Ns} / \mathrm{m}^{5 / 2}\right]$ & $8.96 \cdot 10^{4}$ & & & \\
\hline Sphere radius $[\mathrm{cm}]$ & 4.3 & & & \\
\hline
\end{tabular}

Table 2: States and controls of the optimal control problem depending on the regularisation term used: minimise joint jerk, minimise torque change, or minimise both at the same time.

\begin{tabular}{|l|l|l|l|}
\cline { 2 - 4 } \multicolumn{1}{c|}{} & Minimise joint jerk & Minimise torque change & $\begin{array}{l}\text { Minimise joint jerk and } \\
\text { torque change }\end{array}$ \\
\hline States & $\begin{array}{l}\text { Joint coordinates } \\
\text { Joint velocities } \\
\text { Joint accelerations }\end{array}$ & $\begin{array}{l}\text { Joint coordinates } \\
\text { Joint velocities } \\
\text { Joint torque }\end{array}$ & $\begin{array}{l}\text { Joint coordinates } \\
\text { Joint velocities } \\
\text { Joint accelerations } \\
\text { Joint torque }\end{array}$ \\
\hline Controls & $\begin{array}{l}\text { Joint jerk } \\
\text { Ground reactions }\end{array}$ & $\begin{array}{l}\text { Joint accelerations } \\
\text { Joint torque change } \\
\text { Ground reactions }\end{array}$ & $\begin{array}{l}\text { Joint jerk } \\
\text { Joint torque change } \\
\text { Ground reactions }\end{array}$ \\
\hline
\end{tabular}


Table 3: Values of stride length and swing duration considered in each variation of speed for the four-point alternating gait pattern. Stride length and swing duration were known values in the problem formulation, and speed was computed from them.

\begin{tabular}{|l|c|c|c|c|c|}
\hline & Initial & \multicolumn{2}{|c|}{ Low speed (-15\%) } & \multicolumn{2}{c|}{ High speed (+15\%) } \\
\hline Speed [m/s] & 0.175 & \multicolumn{2}{|c|}{0.15} & \multicolumn{2}{c|}{0.20} \\
\hline Stride length [m] & 0.84 & 0.74 (low) & 0.84 (initial) & 0.95 (high) & 0.84 (initial) \\
\hline Swing duration [s] & 0.96 & 0.96 (initial) & 1.1 (high) & 0.96 (initial) & 0.82 (low) \\
\hline
\end{tabular}

Table 4: Number of iterations, computation time, and mean RMSE of coordinates, mean difference in ROM, and mean RMSE of ground reactions with respect to the dynamically consistent reference data. Coordinates are grouped for torso (lumbar joint), upper limbs (shoulder, elbow and wrist), and lower limbs (hip, knee, ankle, subtalar and metacarpal). The best result of the six initial cost functions for each magnitude is highlighted in bold text. In the last columns, results for the multi-term cost function are shown. It was minimisation of lumbar and lower body mechanical power, upper and lower limbs angular momentum, joint jerk (0.01 weight) and torque change ( 0.01 weight). In bold and underlined, results that are better than all the six initial cost functions are highlighted; in bold and italics, results that are better than the mean of all the six initial cost functions are highlighted.

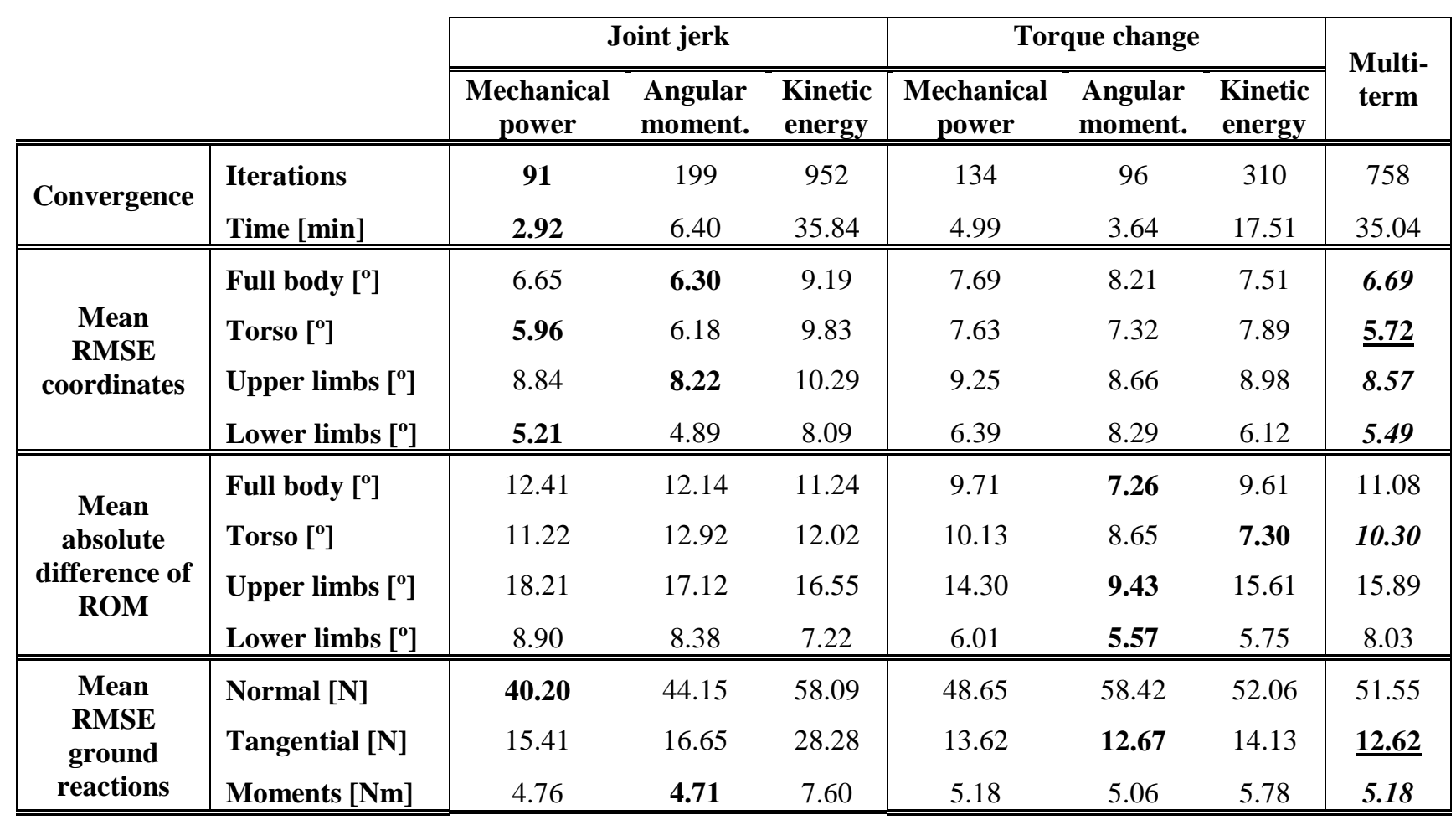




\section{FIGURES}

Figure 1: Left: The healthy subject during the experimental capture. Centre: The 3D OpenSim torque-driven model of the subject and crutches. Right: Locations (red crosses) of the springdamper units of the foot-ground contact models. Numbers indicate each group of spring-damper units (with same coordinate value in medio-lateral direction).
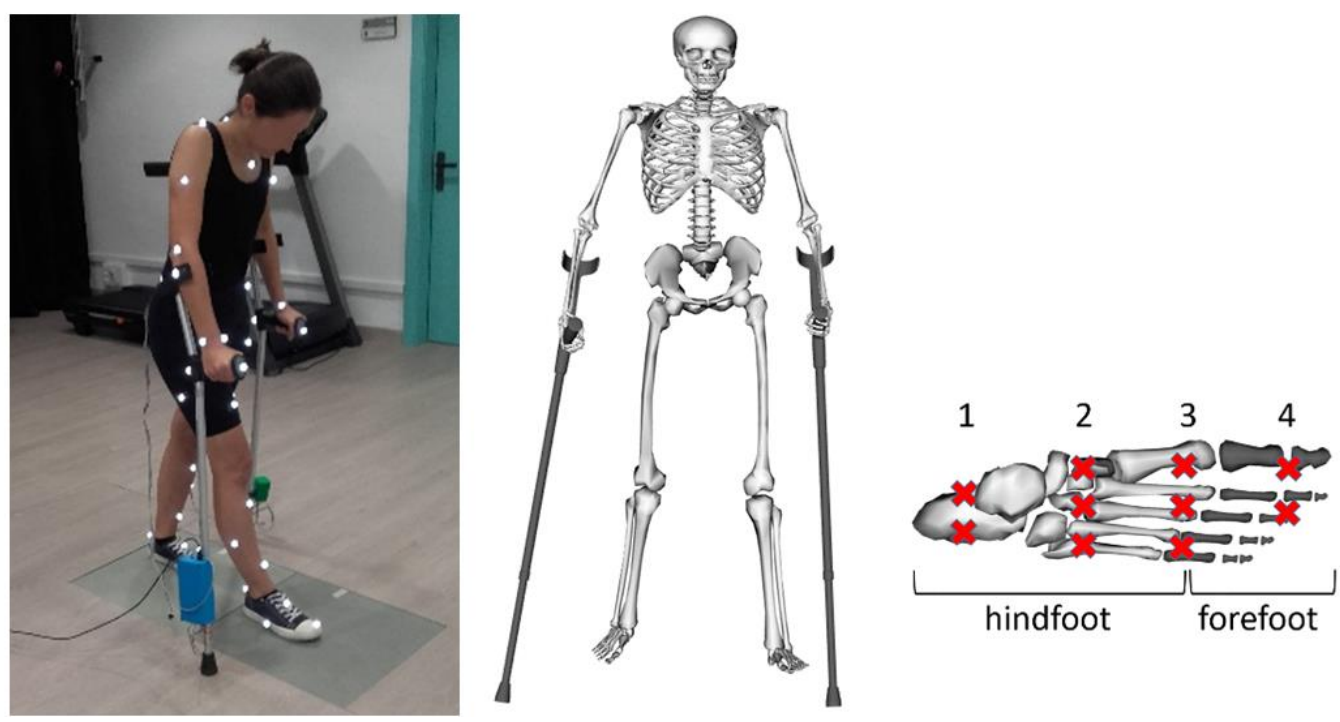
Figure 2: Diagram of the steps followed in this study, including information about the methods and the predictions performed. Top box: Model development and reference data generation. Middle box: Initial problem formulation and comparison of cost functions when predicting a fourpoint non-alternate crutch walking pattern. Bottom box: New crutch walking pattern predictions (four-point alternating, two-point and swing-through), one of them (four-point alternate) at different speeds. Tables and figures showing methods are highlighted in italics, and tables and figures showing results are highlighted in bold. DC stands for "dynamically consistent".

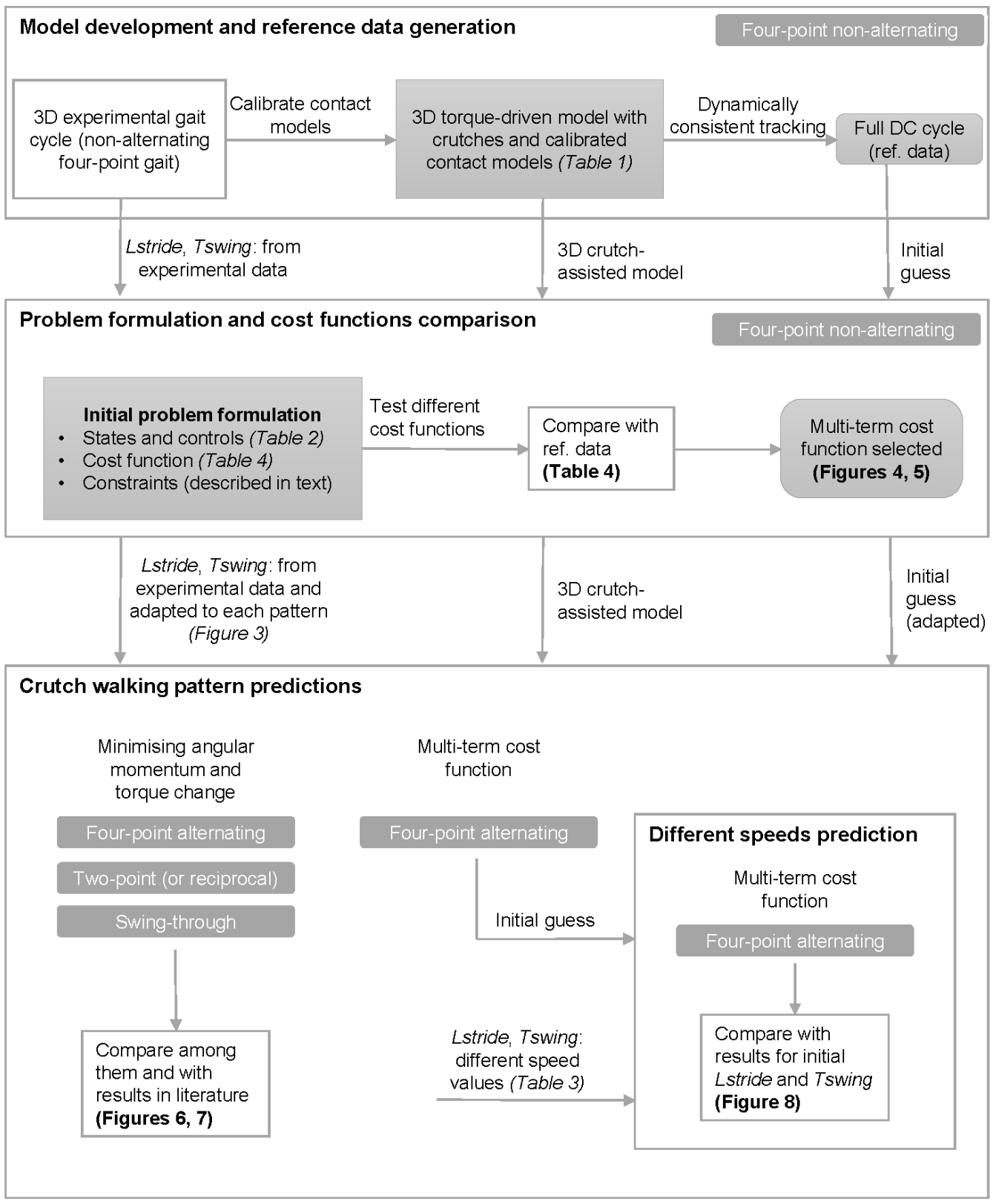


Figure 3: Description of each crutch-walking pattern using stride length (Lstride) and swing duration (Tswing). Initial position was the same for four-point and two-point walking patterns. Multiple support had a duration of a quarter of swing phase duration for four-point gait pattern and of half of the swing phase duration for two-point and swing-through gait patterns. It was split between the beginning and end of the cycle for two-point and swing-through patterns to facilitate convergence and periodicity of ground reactions. The duration of a complete gait cycle was five times swing duration for the four-point gait pattern, and three times swing duration for the two-point and swing-through gait patterns.

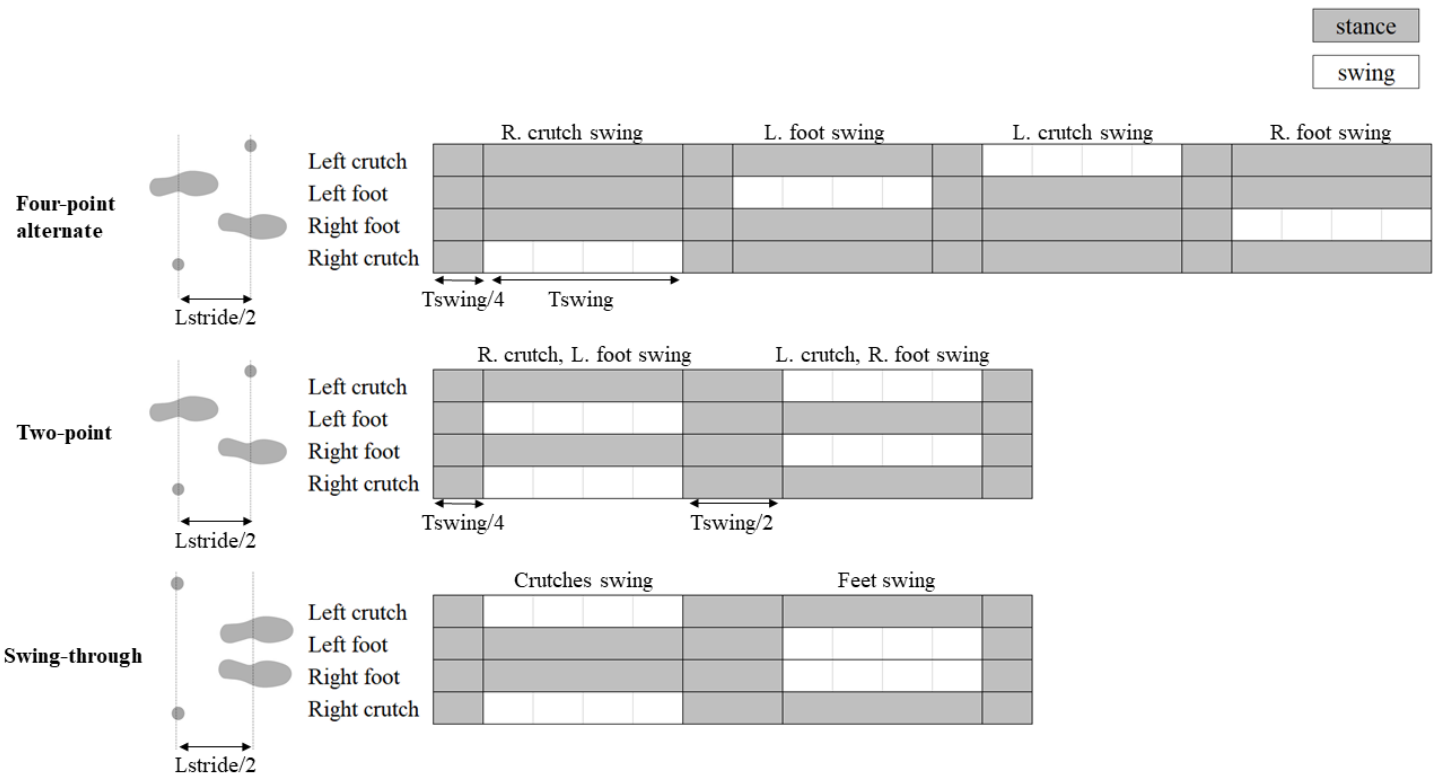

Figure 4: Joint coordinates for the multi-term cost function (minimisation of lumbar and lower body mechanical power, upper and lower limbs angular momentum, joint jerk, and torque change). Predicted curves are shown in solid red lines, and reference curves are shown in dashed blue lines. Note that scales of vertical axes are different for each coordinate. Only the right side of the body is shown. Tracking errors were comparable on the left side.

Figure 5: Foot- and crutch-ground reactions for the multi-term cost function (minimisation of lumbar and lower body mechanical power, upper and lower limbs angular momentum, joint jerk and torque change). Moments are computed at the origin of the calcaneous body. Results are shown in red, and reference values are shown in dashed blue. Note that scales of vertical axes are different for each force or moment. Only the right reactions (foot and crutch) are shown.
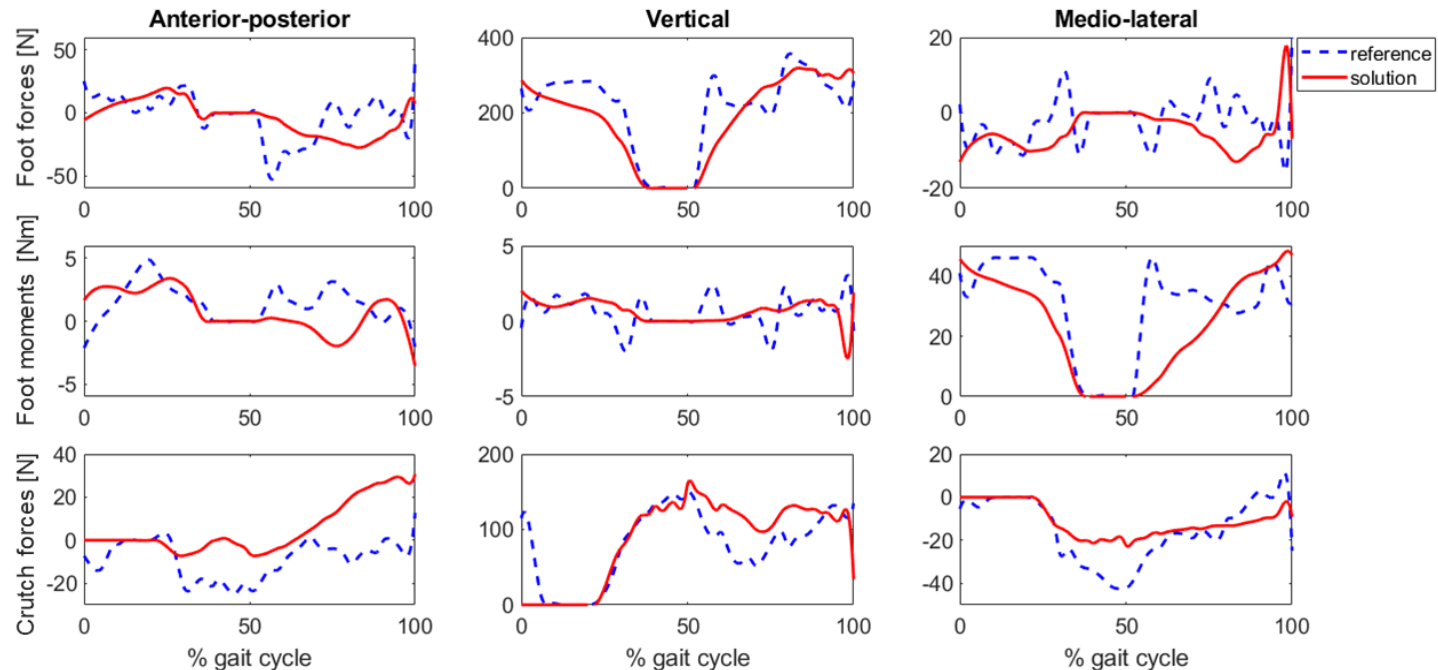

Figure 6: Lateral views and normal ground reactions for three different walking patterns. The cost function was minimising angular momentum and torque change. Normal reactions are shown in \%BW (i.e., percentage of body weight).

Four-point alternate
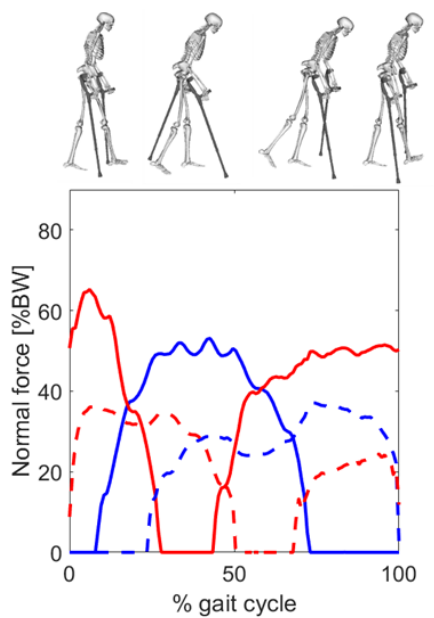

Two-point
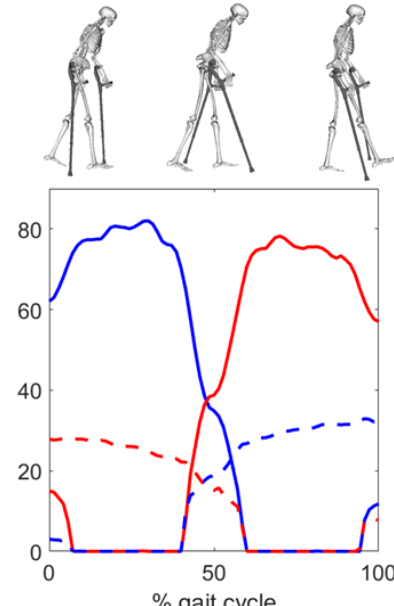

Swing-through
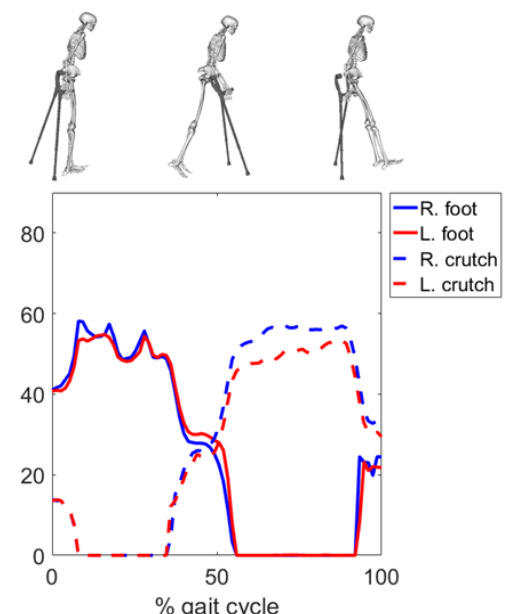
Figure 7: Shoulder coordinates in degrees and torques in \%BW*H (i.e., percentage of body weight times height) for each walking pattern. In blue, results for right side and in red, results for left side. In black dashed lines, maximum and minimum values from experimental studies have been taken for comparison. Values for four-point gait pattern were taken from [6] (only angles), and values for two-point and swing-through gait patterns were taken from [5] (angles and torques). Values for shoulder angles are positive for flexion and adduction.


Figure 8: Some joint coordinates for predictions at different speeds for four-point alternating pattern. All simulations were done with the same swing duration (0.96 s). In blue, right coordinates, in red, left coordinates. Torso tilt is the torso inclination (sum of pelvis tilt and lumbar extension), positive backwards.



\section{REFERENCES}

1. Singh, A., Tetreault, L., Kalsi-Ryan, S., Nouri, A., Fehlings, M.G.: Global prevalence and incidence of traumatic spinal cord injury. Clin. Epidemiol. 6, 309-331 (2014). https://doi.org/10.2147/CLEP.S68889

2. World Health Organization: WHO Global disability action plan 2014-2021: Better health for all people with disability. (2015)

3. Haubert, L.L., Gutierrez, D.D., Newsam, C.J., Gronley, J.K., Mulroy, S.J., Perry, J.: A Comparison of Shoulder Joint Forces During Ambulation With Crutches Versus a Walker in Persons With Incomplete Spinal Cord Injury. (2006). https://doi.org/10.1016/j.apmr.2005.07.311

4. Westerhoff, P., Graichen, F., Bender, A., Halder, A., Beier, A., Rohlmann, A., Bergmann, G.: Clinical Biomechanics In vivo measurement of shoulder joint loads during walking with crutches. JCLB. 27, 711-718 (2012). https://doi.org/10.1016/j.clinbiomech.2012.03.004

5. Perez-Rizo, E., Trincado-Alonso, F., Pérez-Nombela, S., Del Ama-Espinosa, A., JiménezDíaz, F., Lozano-Berrio, V., Gil-Agudo, A.: Application of a model to analyze shoulder biomechanics in adult patients with spinal cord injury when walking with crutches in two different gait patterns. NeuroRehabilitation. 40, 129-140 (2017). https://doi.org/10.3233/NRE-161398

6. Fischer, J., Nüesch, C., Göpfert, B., Mündermann, A., Valderrabano, V., Hügle, T.: Forearm pressure distribution during ambulation with elbow crutches : a cross-sectional study. 19 (2014). https://doi.org/10.1186/1743-0003-11-61

7. Miller, L.E., Zimmermann, A.K., Herbert, W.G.: Clinical effectiveness and safety of powered exoskeleton-assisted walking on SCI patients. 455-466 (2016). https://doi.org/10.2147/MDER.S103102

8. Louie, D.R., Eng, J.J., Lam, T.: Gait speed using powered robotic exoskeletons after spinal cord injury: a systematic review and correlational study. J. Neuroeng. Rehabil. 12, 82-92 (2015). https://doi.org/10.1186/s12984-015-0074-9

9. Lancini, M., Bodini, I., Sansoni, G.: UPPER LIMB LOADS DURING ROBOTIC ASSISTED GAIT : A MEASURING SYSTEM TO Conference Paper · October 2017. (2017). https://doi.org/10.1142/9789813231047

10. Behrman, A.L., Ardolino, E., Vanhiel, L.R., Kern, M., Atkinson, D., Lorenz, D.J., Harkema, S.J.: Assessment of functional improvement without compensation reduces variability of outcome measures after human spinal cord injury. Arch. Phys. Med. Rehabil. 93, 15181529 (2012). https://doi.org/10.1016/j.apmr.2011.04.027

11. Liu, G., Zhang, Y., Xie, S.-Q.Q., Xue, A.: Optimal control and biomechanics of ambulation with spring-loaded crutches. Int. J. Adv. Robot. Syst. 8, 1-11 (2011). https://doi.org/10.5772/10664

12. Ackermann, M., Taissun, B.A.: A computational study of the swing phase of the gait with standard and spring-loaded crutches. Proc. IEEE RAS EMBS Int. Conf. Biomed. Robot. Biomechatronics. 1476-1481 (2012). https://doi.org/10.1109/BioRob.2012.6290718

13. Tashman, S., Zajac, F.E., Perkash, I.: Modeling and simulation of paraplegic ambulation in a reciprocating gait orthosis. J. Biomech. Eng. 117, 300-308 (1995).

https://doi.org/10.1115/1.2794185

14. Fournier, B.N., Lemaire, E.D., Smith, A.J.J., Doumit, M.: Modeling and Simulation of a Lower Extremity Powered Exoskeleton. IEEE Trans. Neural Syst. Rehabil. Eng. 26, 15961603 (2018). https://doi.org/10.1109/TNSRE.2018.2854605

15. Mouzo, F., Lugris, U., Pamies-Vila, R., Cuadrado, J., Lugrís, U., Pamies-Vila, R., Cuadrado, J.: Skeletal-level control-based forward dynamic analysis of acquired healthy and assisted 
gait motion. Multibody Syst. Dyn. 44, (29) (2018). https://doi.org/10.1007/s11044-01809634-4

16. van der Spek, J.H., Veltink, P.H., Hermens, H.J., Koopman, B.F.J.M., Boom, H.B.K.: A model-based approach to stabilizing crutch supported paraplegic standing by artificial hip joint stiffness. IEEE Trans. Neural Syst. Rehabil. Eng. 11, 443-451 (2003). https://doi.org/10.1109/TNSRE.2003.819939

17. Font-Llagunes, J.M., Barjau, A., Pàmies-Vilà, R., Kövecses, J.: Dynamic analysis of impact in swing-through crutch gait using impulsive and continuous contact models. Multibody Syst. Dyn. 28, 257-282 (2012). https://doi.org/10.1007/s11044-011-9300-9

18. Meyer, A.J., Eskinazi, I., Jackson, J.N., Rao, A. V., Patten, C., Fregly, B.J.: Muscle synergies facilitate computational prediction of subject-specific walking motions. Front. Bioeng. Biotechnol. 4, 77 (2016). https://doi.org/10.3389/fbioe.2016.00077

19. Fregly, B.J., Reinbolt, J.A., Rooney, K.L., Mitchell, K.H., Chmielewski, T.L.: Design of patient-specific gait modifications for knee osteoarthritis rehabilitation. IEEE Trans. Biomed. Eng. 54, 1687-1695 (2007). https://doi.org/10.1109/TBME.2007.907637

20. Esposito, E.R., Miller, R.H.: Maintenance of muscle strength retains a normal metabolic cost in simulated walking after transtibial limb loss. PLoS One. 13, 1-19 (2018). https://doi.org/10.1371/journal.pone.0191310

21. Sreenivasa, M., Millard, M., Felis, M.L., Mombaur, K., Wolf, S.I.: Optimal control based stiffness identification of an ankle-foot orthosis using a predictive walking model. Front. Comput. Neurosci. 11, (13) (2017)

22. Mombaur, K., Ho Hoang, K.L.: How to best support sit to stand transfers of geriatric patients: Motion optimization under external forces for the design of physical assistive devices. J. Biomech. 58, 131-138 (2017). https://doi.org/10.1016/j.jbiomech.2017.04.037

23. Sardini, E., Serpelloni, M., Lancini, M.: Wireless instrumented crutches for force and movement measurements for gait monitoring. IEEE Trans. Instrum. Meas. 64, 3369-3379 (2015). https://doi.org/10.1109/TIM.2015.2465751

24. Rajagopal, A., Dembia, C., DeMers, M., Delp, D., Hicks, J.L., Delp, S.: Full body musculoskeletal model for muscle-driven simulation of human gait. IEEE Trans. Biomed. Eng. 9294, 1-1 (2016). https://doi.org/10.1109/TBME.2016.2586891

25. Jackson, J.N., Hass, C.J., Fregly, B.J.: Development of a subject-specific foot-ground contact model for walking. J. Biomech. Eng. (in press), 091002 (2016). https://doi.org/10.1115/1.4034060

26. Hunt, K., Crossley, E., Hunt, K.H., Crossley, F.R.E.: Coefficient of restitution interpreted as damping in vibroimpact. https://doi.org/10.1115/1.3423596ï

27. Febrer-Nafría, M., Pallarès-López, R., Font-Llagunes, J.M.: Calibration of foot-ground and crutch-ground contact models for optimal control prediction of crutch-assisted walking motions. In: ECCOMAS Thematic Conference on Multibody Dynamics. p. (2 pages). , Duisburg (Germany)

28. Van Den Bogert, A.J., Blana, D., Heinrich, D.: Implicit methods for efficient musculoskeletal simulation and optimal control. Procedia IUTAM. 2, 297-316 (2011). https://doi.org/10.1016/j.piutam.2011.04.027

29. Rao, A. V., Benson, D. a., Darby, C., Patterson, M., Francolin, C., Sanders, I., Huntington, G.T.: GPOPS- II: A MATLAB software for solving multiple-phase optimal control problems using hp-adaptive gaussian quadrature collocation methods and sparse nonlinear programming. ACM Trans. Math. Softw. 37, 1-39 (2014). https://doi.org/10.1145/1731022.1731032

30. Dorschky, E., Nitschke, M., Seifer, A.K., van den Bogert, A.J., Eskofier, B.M.: Estimation of 
gait kinematics and kinetics from inertial sensor data using optimal control of musculoskeletal models. J. Biomech. 95, 109278 (2019). https://doi.org/10.1016/j.jbiomech.2019.07.022

31. Kaphle, M., Eriksson, A.: Optimality in forward dynamics simulations. J. Biomech. 41, 1213-1221 (2008). https://doi.org/10.1016/j.jbiomech.2008.01.021

32. Koch, M.W., Ringkamp, M., Leyendecker, S.: Discrete Mechanics and Optimal Control of Walking Gaits. J. Comput. Nonlinear Dyn. 12, 021006 (2016). https://doi.org/10.1115/1.4035213

33. Xiang, Y., Arora, J.S., Abdel-Malek, K.: Physics-based modeling and simulation of human walking: a review of optimization-based and other approaches. Struct. Multidiscip. Optim. 42, 1-23 (2010). https://doi.org/10.1007/s00158-010-0496-8

34. Fluit, R., van der Krogt, M.M., van der Kooij, H., Verdonschot, N., Koopman, H.F.J.M.: A simple controller for the prediction of three-dimensional gait. J. Biomech. 45, 2610-2617 (2012). https://doi.org/10.1016/j.jbiomech.2012.08.019

35. Ren, L., Jones, R.K., Howard, D.: Predictive modelling of human walking over a complete gait cycle. J. Biomech. 40, 1567-1574 (2007). https://doi.org/10.1016/j.jbiomech.2006.07.017

36. Herr, H., Popovic, M.: Angular momentum in human walking. J. Exp. Biol. 211, 467-481 (2008). https://doi.org/10.1242/jeb.008573

37. Felis, M.L., Mombaur, K.: Modeling and optimization of human walking. In: Mombaur, K. and Berns, K. (eds.) Modeling, Simulation and Optimization. pp. 31-42 (2013)

38. Lane, P.L., LeBlanc, R.: Crutch walking. Orthop. Nurs. 9, 31-8

39. Ezati, M., Ghannadi, B., McPhee, J.: A review of simulation methods for human movement dynamics with emphasis on gait. Multibody Syst. Dyn. (2019). https://doi.org/10.1007/s11044-019-09685-1

40. Miller, R.H.: A comparison of muscle energy models for simulating human walking in three dimensions. J. Biomech. 47, 1373-1381 (2014). https://doi.org/10.1016/j.jbiomech.2014.01.049

41. Umberger, B.R., Miller, R.H.: Optimal Control Modeling of Human Movement. https://doi.org/10.1007/978-3-319-30808-1

42. UNO, Y.: Formation and Control of Optical Trajectory in Human Multi-joint Arm Movement-Minimim Torque-change Model. Biol. Cybern. 61, 89-101 (1989)

43. Nakano, E., Imamizu, H., Osu, R., Uno, Y., Gomi, H., Yoshioka, T., Kawato, M.: Quantitative Examinations of Internal Representations for Arm Trajectory Planning: Minimum Commanded Torque Change Model. J. Neurophysiol. 81, 2140-2155 (2017). https://doi.org/10.1152/jn.1999.81.5.2140

44. Wada, Y., Kaneko, Y., Nakano, E., Osu, R., Kawato, M.: Quantitative examinations for multi joint arm trajectory planning - Using a robust calculation algorithm of the minimum commanded torque change trajectory. Neural Networks. 14, 381-393 (2001). https://doi.org/10.1016/S0893-6080(01)00026-0

45. Lee, J. U., Kim, M. Y., Kim, J. H., Lee, J. A., Yoon, N. M., Hwang, B. Y., Kim, J.: Analysis of Plantar Foot Pressure during the Non- crutch , Two-point , and Four-point Crutch Gait. Gait Posture. 489-493 (2011)

46. Perry, A.G., Potter, P.A., Ostendorf, W.: Nursing interventions \&amp; clinical skills.

47. Noreau, L., Richards, C.L., Comeau, F., Tardif, D.: IN PARAPLEGIC ANALYSIS OF SWINGTHROUGH GAIT AND NON-DISABLED INDIVIDUALS. (1995) 
48. Schulz, B.W., Ashton-Miller, J.A., Alexander, N.B.: The effects of age and step length on joint kinematics and kinetics of large out-and-back steps. Clin. Biomech. 23, 609-618 (2008). https://doi.org/10.1016/J.CLINBIOMECH.2008.01.006

49. Berret, B., Chiovetto, E., Nori, F., Pozzo, T.: Evidence for composite cost functions in arm movement planning: An inverse optimal control approach. PLoS Comput. Biol. 7, 1-10 (2011). https://doi.org/10.1371/journal.pcbi.1002183

50. Mombaur, K., Truong, A., Laumond, J.P.: From human to humanoid locomotion-an inverse optimal control approach. Auton. Robots. 28, 369-383 (2010). https://doi.org/10.1007/s10514-009-9170-7

51. Font-Llagunes, J.M., Lugrís, U., Clos, D., Alonso, F.J., Cuadrado, J.: Design, control and pilot study of a lightweight and modular robotic exoskeleton for walking assistance after spinal cord injury. J. Mech. Robot. 12, 031008 (8 pp.) (2020)

52. Gil-Agudo, A., Pérez-Rizo, E., Del Ama-Espinosa, A., Crespo-Ruiz, B., Pérez-Nombela, S., Sánchez-Ramos, A., Pàmies-Vilà, R., Sánchez-Ramos, A.: Comparative biomechanical gait analysis of patients with central cord syndrome walking with one crutch and two crutches. Clin. Biomech. 24, 551-557 (2009). https://doi.org/10.1016/j.clinbiomech.2009.04.009

53. Freddolini, A.M., Esposito, F., Corvi, A., Braccio, P., Latella, L.: Does crutch length influence gait parameters after total hip replacement surgery ? Gait Posture. (2017). https://doi.org/10.1016/j.gaitpost.2017.07.035

54. Segura, A., Piazza, S.J.: Mechanics of Ambulation With Standard and Spring-Loaded Crutches. Arch. Phys. Med. Rehabil. 88, 1159-1163 (2007). https://doi.org/10.1016/j.apmr.2007.05.026

55. Zhang, Y., Liu, G., Xie, S., Liger, A.: Biomechanical Evaluation of an Innovative SpringLoaded Axillary Crutch Design. Assist. Technol. 23, 225-231 (2011).

https://doi.org/10.1080/10400435.2011.614676 